\title{
Acknowledgement of our reviewers and update of the journal
}

\author{
Diana Epstein ${ }^{1}(1) \cdot$ Antonia M. Joussen $^{2} \cdot$ Taiji Sakamoto $^{3} \cdot$ Srinivas Sadda $^{4}$ \\ Published online: 9 February 2022 \\ ๑ The Author(s), under exclusive licence to Springer-Verlag GmbH Germany, part of Springer Nature 2022
}

As we navigate ourselves out of 2021 and into 2022 with the hope that life will be able to develop into a new "normal," some changes have taken place in the publishing world which has resulted in this year's acknowledgement to reviewers looking quite different to previous years.

With the introduction of GDPR (Gender Data Protection Regulation) [1], we are no longer able to publish the names of all reviewers who have assisted with reviews for the journal; hence, we would like to thank all reviewers, and those who were mentored by our senior reviewers to review for the journal.

The publisher will be happy to prepare and send certificates of acknowledgement to reviewers. As this is not automated, please do contact the editorial office and we will be happy to forward your details to the publisher for preparation of certificates.

\section{Mentorship team}

We are very proud of our pro-active editorial board and in 2020, the mentorship team was founded [2]. This team initially consisted of 3 pro-active editorial board members and from January this year, we are pleased to advise that the team has increased to 9 members. During 2021 and due to COVID, the Editors felt it was not feasible to process papers via the mentorship team but we believe the team will be kept busy from 2022 onwards.

The team names can be found on the back cover of the printed issue and online. This will result in being able to offer mentorship opportunities not just to former Eastern

Diana Epstein

graefes_archive@di-ep.com

1 Di-Ep Biomedical Editorial Services Ltd, Glasgow, Scotland, UK

2 Augenklinik der Charite, Berlin, Germany

3 Kagoshima University, Kagoshima, Japan

4 Doheny Eye Institute, Los Angeles, CA, USA countries but also to authors based in lower-middle-income countries (LMIC).

All papers will be processed as usual and the Editors will identify the papers they feel could benefit from input from the mentorship team. This is to help junior authors from LMICs to publish papers and in the case of acceptance, a note will be placed that assistance from the mentorship team was received for the paper.

\section{Social Media (SoMe)}

During 2021, there has been an increase with Social Media (SoMe) activities. We have a dedicated SoMe team of board members and we are happy to note that some additional board members have joined the team. We are also indebted to the German Society of Ophthalmology (DOG) and Edmund Tsai from UCLA for their support with retweeting and commenting on posts we tweet and also to the many followers who support us with "liking," "retweeting," and "commenting" on our posts.

The most liked tweets in 2020-the most liked cover image (Fig. 1) and most liked post (Fig. 2).

In the altmetrics, 3 of the top 5 papers were high scoring and dealt with COVID (Table 1).

Please do follow @diepbiomed and @ClinMed Journal.

\section{Mini-reviews}

A new article type was recently introduced. In essence, they are short reviews tackling the "big questions" currently facing various different sub-divisions of the field of ophthalmology.

We have observed a dramatic rise in the overall number of publications in the field that has mirrored an exponential growth in our knowledge and understanding of eye disease and research. With this vast array of articles, it has become increasingly difficult for readers to interpret the key messages and major findings, particularly as articles may feature 
Fig. 1 Most liked cover image and Most liked tweet

Diana Epstein @diepbiomed

Apr 27, 2020

May cover from rdcu.be/b3M7k @KennethLi56 protect ophthalmologists \#COVID-19 transmission @ClinMedJournals @SpringerSurgery @Ophthalmologen @EdmundTsuiMD @MalocaPeter @meyer_professor @SakamotoTaiji @AndrzejGrzybow @oliverzeitz @eyedoctorsIRL @iscev_org @OpEyesight

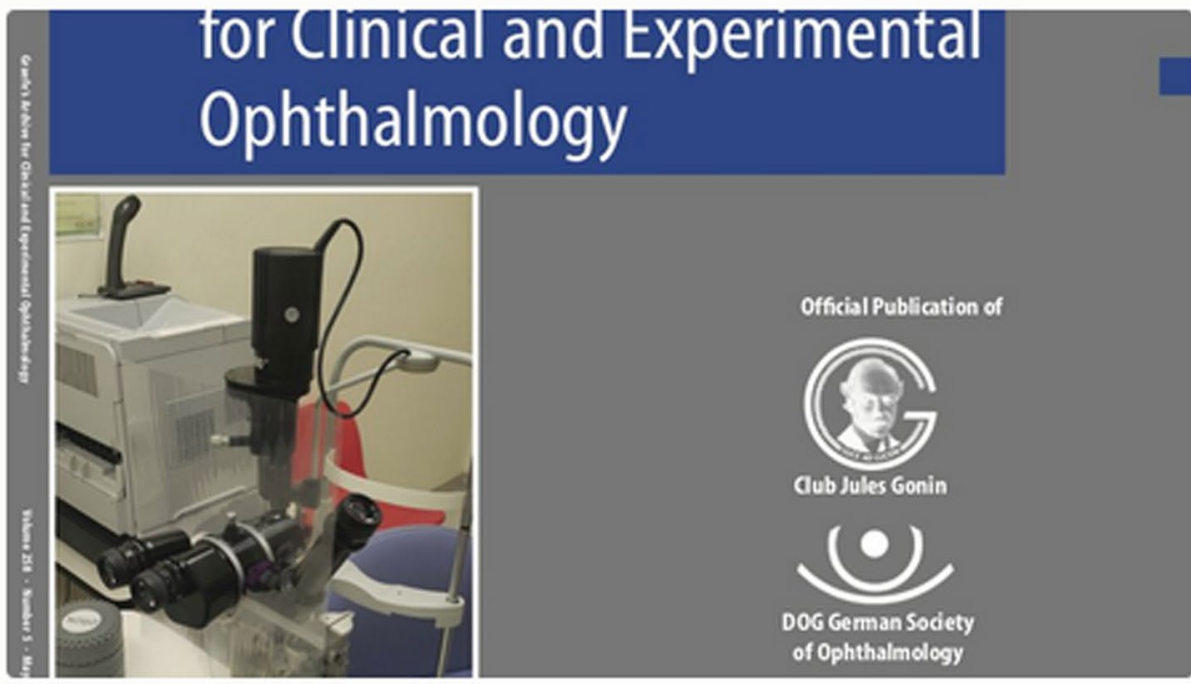

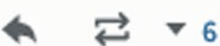
$9[4] \rightarrow \ldots$

Diana Epstein @diepbiomed Nov 2, 2020

Graefes November highlight rdcu.be/b833N Multicolor imaging in MacTel @ClinMedJournals @Ophthalmologen @meyer_professor @oliverzeitz @AndrzejGrzybow @EdmundTsuiMD @SakamotoTaiji @SydneySaveSight @fightblindness
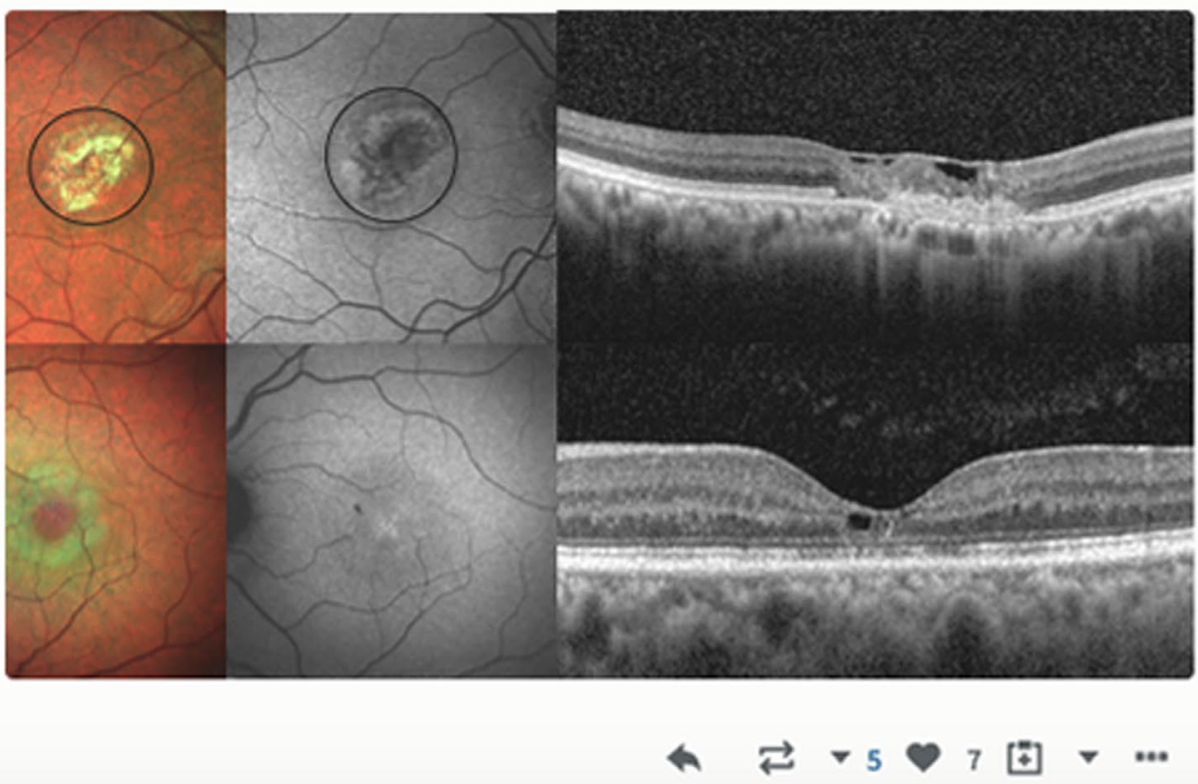
Table 1 Altmetrics

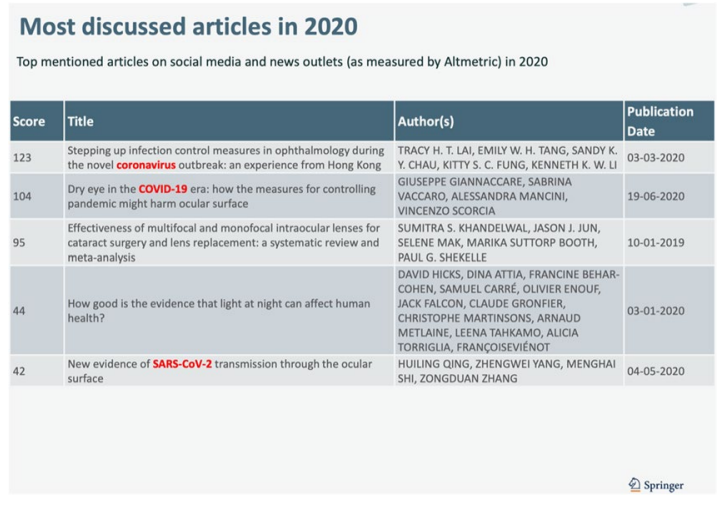

inconsistent or conflicting information. This has highlighted the importance of review articles which can aggregate and harmonize the information into a readily consumable format for readers. Major review articles, however, are significant undertakings and they can quickly go out of date, given the rapid pace of scientific and clinical advance.

Articles can cover "hot topics" for example: artificial intelligence, updates on new therapeutics, new insights into disease mechanisms/pathology/diagnostic techniques to name but a few.

The Editors are looking for mini-reviews as a short overview-word limit of 4000 words of a specific topic which has novelty and can help shape the practice of ophthalmology or provide new questions to stimulate future research directions.

\section{Topical collections}

We currently have 4 active online topical collections [3] for which we would like to receive submissions. The authors are invited to submit and note on the cover letter that you would like your paper to be considered. We currently have as follows:

Ophthalmic Plastic Surgery

Breakthroughs in Artifical Intelligence (AI) for ophthalmology

Macular Holes

Perspectives on COVID-19

Finally, we would like to thank our publisher Patrizia Bianchi at Springer Nature for her support of the journal and all reviewers and readers for your support of the journal during the past year and hopefully a healthy year will be ahead.

\section{References}

1. General Data Protection Regulation (GDPR)-official legal text https://gdpr-info.eu Accessed on 3 Jan 2022

2. Epstein D, Kozak I (2020) The delicate topic of mentorship Graefe's Archive for. Clin Experiment Ophthalmol 258:1. https:// doi.org/10.1007/s00417-019-04518-5

3. https://link.springer.com/journal/417/collections date of access 13 January 2022

Publisher's note Springer Nature remains neutral with regard to jurisdictional claims in published maps and institutional affiliations. 